\title{
HACIA UNA INTERPRETACIÓN FÍSICO-CAUSAL DE LA INFORMACIÓN EN CONTEXTOS COMUNICACIONALES
}

\author{
Cristian Ariel López \\ Universidad de Buenos Aires \\ Université de Lausanne \\ Consejo Nacional de Investigaciones Científicas de Argentina (CONICET) \\ lopez.cristian1987@gmail.com \\ OLIMPIA IRIS LOMBARDI \\ Universidad de Buenos Aires \\ Consejo Nacional de Investigaciones Científicas de Argentina (CONICET) \\ olimpiafilo@gmail.com
}

RESUMEN: El objetivo del presente artículo es proponer una nueva interpretación del concepto de información en contextos comunicacionales: una interpretación físicocausal. Apelando a las teorías manipulabilistas de la causación, principalmente en su versión intervencionista, buscaremos mostrar que la información comunicacional es una propiedad física que podemos manipular para generar situaciones comunicacionales. Este enfoque nos permite entender la naturaleza de la comunicación como estructura causal, puesta de manifiesto mediante criterios manipulabilistas. Confiamos en que el enfoque propuesto logra superar ciertas dificultades de las interpretaciones de la información actualmente vigentes en la literatura.

PALABRAS CLAVE: comunicación, manipulabilismo, información estadística, causación, filosofía de la física

SUMMARY: The aim of this paper is to offer a new interpretation of information: a causal-physical interpretation. We shall turn to manipulability theories of causation (mainly to its interventionist version) in order to show that "information" refers to a physical property, which we can manipulate to bring about communication situations. According to our approach, the nature of communication is a causal structure, unveiled by manipulability criteria. Furthermore, we think that our approach will be able to deal with some issues of present-day interpretations of information.

KEY WORDS: communication, manipulability, statistical information, causation, philosophy of physics

\section{Introducción}

Como consecuencia de la fuerte presencia actual de la noción de información tanto en la vida cotidiana como en el mundo científico, 
la reflexión acerca de su significado y funciones ha ingresado decididamente en el ámbito de la filosofía de la ciencia, en particular, de la filosofía de la física. Sin embargo, apenas nos abocamos a dicha reflexión, hallamos curiosamente que, lejos de tratarse de un concepto de fácil comprensión, lidiamos con una noción sumamente oscura y confusa, probablemente debido a su ubicua presencia. No existe en la comunidad científica y filosófica consenso acerca de cuál es la naturaleza de la información: no parece haber un concepto unificado bajo el cual sea posible reconducir las múltiples y variadas concepciones. Parece verosímil que el motivo radique en que, efectivamente, la naturaleza del concepto es sumamente polimórfica y polisemántica, como bien señala Luciano Floridi (2011). El surgimiento de la filosofía de la información, como campo de indagación sistemático y autónomo, da cuenta de la relevancia filosófica que el concepto ha adquirido en los últimos años.

Un primer paso para abrirse paso a través de este tupido y laberíntico campo consiste en atender a las teorías formales de la información usualmente utilizadas en diferentes teorías científicas y en el ámbito tecnológico. Particular atención ha recibido la teoría matemática de la comunicación de Claude Shannon (1948) (debido a su rotundo éxito tecnológico, junto con su capacidad y simplicidad formal), que se ha convertido en el punto de partida usual de cualquier indagación filosófica. Pero, como afirman Olimpia Lombardi, Sebastian Fortin y Leonardo Vanni (2015), el formalismo de Shannon por sí solo no es suficiente para obtener un concepto sustantivo del término información. De hecho, tal formalismo es compatible con diversas interpretaciones que conducen a escenarios muy diversos. En filosofía de la información y en filosofía de la física, comúnmente se distinguen tres interpretaciones genéricas: una interpretación física (Landauer 1991, 1996, Rovelli 1996, Stonier 1996), una interpretación epistémica (Dretske 1981, MacKay 1956, 1969) y una deflacionista (Timpson 2004, 2013).

Si bien la teoría de la información de Shannon es compatible con cualquiera de estas interpretaciones, todas ellas sufren diversas dificultades a la hora de dar cuenta de situaciones concretas donde el concepto de información está involucrado. El objetivo de este artículo es señalar tales dificultades y sentar las bases con miras a una interpretación de la información en el ámbito de la filosofía de la física que logre superarlas. En particular, propondremos una interpretación físico-causal de la información, según la cual la información es fundamentalmente una propiedad física que es posible manipular para 
generar situaciones comunicacionales. De acuerdo con nuestro enfoque, los contextos comunicacionales exigen estructuras causales que se ponen al descubierto mediante técnicas de intervención y control. Para respaldar esta idea, utilizaremos la teoría manipulabilista de la causación desarrollada por James Woodward $(2003,2007)$ y Judea Pearl (2000).

\section{Información comunicacional: poniendo el concepto} de información en perspectiva

\section{1. ¿Qué es información?}

Intuitivamente, el concepto de información parece estar relacionado con la transmisión y el almacenamiento de datos. Luciano Floridi (2017) destaca este punto y ofrece una definición de la información basada en la noción de dato. De acuerdo con Floridi, $\sigma$ es una instancia de información si y sólo si $\sigma$ está conformada por uno o más datos, y si estos datos están bien formados. Floridi también señala que, si esos datos son significativos, entonces $\sigma$ es una instancia de información semántica (Floridi 2017). Si bien esta definición es de ayuda para aproximarnos al concepto de información, es necesario introducir más precisiones y distinciones debido al amplio abanico de definiciones y aplicaciones posibles del término. El propio Claude Shannon advertía esta situación cuando afirmaba:

La palabra "información" ha recibido diferentes significados por varios autores en el campo general de la teoría de la información. Probablemente, un buen número de ellos serán suficientemente útiles en ciertas aplicaciones y merezcan un estudio posterior y un reconocimiento permanente. Difícilmente se podría esperar que un concepto simple de información pudiera dar cuenta, de manera satisfactoria, de las numerosas aplicaciones posibles de este campo general. (Shannon 1993, p. 180; las cursivas son mías.)

Por este motivo, resulta metodológicamente útil y conceptualmente esclarecedor comenzar distinguiendo los diferentes dominios en los que el concepto de información es utilizado, constriñendo de esta manera en qué sentido hablaremos de información a lo largo de este artículo.

En un primer acercamiento sistemático conviene distinguir al menos dos tipos de enfoques: un enfoque semántico y un enfoque estadístico. Según el primero, la información porta contenido semántico e involucra nociones como verdad, significado o representación 
(Bar-Hillel y Carnap 1953, Bar-Hillel 1964, Floridi 2011). Desde esta perspectiva, cuando decimos que "A tiene información acerca de B", decimos que "A significa o representa B". En términos de la definición de información basada en datos, Floridi menciona que este enfoque considera la información fundamentalmente como "dato significativo bien formado" (2016). En cambio, de acuerdo con el enfoque estadístico (mayoritariamente empleado en las ciencias físicas y formales), las propiedades estadísticas de un sistema y/o las correlaciones entre los estados de dos sistemas son los aspectos relevantes a la hora de hablar de información; la información es meramente "dato bien formado" (siguiendo la nomenclatura de Floridi), despojado de cualquier dimensión semántica. Christopher Timpson (2004, 2013) ha denominado información técnica a este sentido de información, y es el que utilizaremos a lo largo de este artículo.

A pesar de haber circunscrito el análisis de la información a un enfoque estadístico y técnico, el concepto todavía sigue siendo poco específico y muy general. Un segundo paso consiste en distinguir contextos donde el término se utiliza comúnmente. Los contextos permiten identificar diferentes aplicaciones del concepto de información estadística de las que se desprenden diferentes propiedades y sentidos más específicos del término. En general, se pueden identificar al menos dos contextos relevantes en filosofía de la física: un contexto computacional y un contexto comunicacional.

Cuando se utiliza el término "información" en un contexto computacional, usualmente se concibe la información como algo que puede ser computado y almacenado de manera eficiente. Por ejemplo, las teorías de la complejidad algorítmica (v.g., Chaitin 1966, Kolmogorov 1965) buscan medir los mínimos recursos necesarios para que un mensaje individual pueda ser reconstruido de manera efectiva. En cambio, en un contexto comunicacional, la información se entiende como algo que se puede transmitir entre dos puntos del espacio con fines comunicativos. La teoría matemática de la comunicación de Claude Shannon (1948) es el ejemplo paradigmático de una teoría formal que asume un enfoque puramente estadístico de la información en un contexto comunicacional. Dado que la discusión científica y filosófica acerca del concepto de información en filosofía de la física ha presupuesto principalmente contextos comunicacionales (utilizando como punto de partida, sobre todo, la teoría matemática de Shannon), en este trabajo nos circunscribiremos exclusivamente a la noción de información comunicacional. 


\section{2. ¿Qué es comunicación?}

Más allá de las complejidades técnicas que una situación comunicacional pueda tener, es posible extraer algunos elementos y características comunes. En general, toda situación comunicacional incluye al menos tres elementos: una fuente, un canal y un destinatario. De manera muy abstracta, la fuente $S$ produce una cantidad de información $I(S)$ que será transmitida al destinatario $D$, el cual recibe una cantidad de información determinada, $I(D)$; la información es transmitida por un canal $C$ que vincula fuente y destinatario. Tanto $S$ como $D$ son sistemas con un rango de estados posibles, cada uno con su propia probabilidad, y es en estos términos en los que se calcula la cantidad de información producida y recibida.

Otro elemento central es la caracterización del canal de comunicación. El canal $C$ se caracteriza por medio de probabilidades condicionales que vinculan la aparición de los estados de la fuente con la aparición de los estados del destinatario. Si bien el éxito de la comunicación depende de que toda la información generada en la fuente, y sólo ésta, pueda ser reproducida en el destinatario (caso de comunicación perfecta), las situaciones comunicacionales reales no suelen lograr tal grado de perfección: la existencia de equivocidad o ruido impiden la posibilidad de una correlación uno-a-uno entre los estados de la fuente y los estados del destinatario. Cuando hay equivocidad, existe información generada por $S$ pero no recibida en $D$; en cambio, cuando hay ruido existe información recibida por $D$ pero que no proviene de $S$. A la información producida por $S$ que efectivamente llega a $D$ habitualmente se le llama información mutua, $I(S ; D)$. Más allá de cómo se calculan estas cantidades, es posible generalizar la siguiente relación:

$$
I(S ; D)=I(S)-E=I(D)-N
$$

Por lo tanto, en situaciones reales de comunicación el objetivo es reducir la cantidad de ruido y de equivocidad al mínimo, sea que se mejoren las características del canal de manera que se evite la pérdida de información, o sea que se utilicen dispositivos que filtren el ruido, incrementando así la cantidad de información mutua.

Desde un punto de vista conceptual, podemos extraer dos notas esenciales de cualquier proceso comunicacional: (a) la propiedad de ser un proceso asimétrico, y (b) la de ser un proceso productivo o un "hacedor de diferencias" ("difference maker"). Con respecto al primer punto, toda situación comunicacional en la que una pieza o instancia de información se transmite involucra al menos dos sistemas 
físicos separados, uno de los cuales envía un mensaje mientras que el otro lo recibe. Si bien desde un punto de vista altamente abstracto una situación comunicacional podría parecer simétrica, ya que dependería únicamente de poder definir correlaciones entre diferentes variables, esto pasa por alto cuál es el objetivo de la comunicación tal como el propio Shannon lo define al introducir su teoría: "El problema fundamental de la comunicación es reproducir en un punto exacta o aproximadamente un mensaje seleccionado en otro punto [...]; el aspecto relevante es que el mensaje real es un mensaje seleccionado de un conjunto de posibles mensajes" (Shannon 1948, p. 379). Por este motivo, meras correlaciones entre las variables de dos sistemas no son suficientes para definir una situación comunicacional: las correlaciones definidas vinculan ciertas variables con ciertas otras variables; y un aspecto esencial de la comunicación es poder identificar esas ciertas variables. En otras palabras, para obtener una comunicación exitosa necesitamos reproducir o identificar en el receptor un mensaje específico que fue seleccionado en la fuente. Este rasgo establece una clara dependencia entre los estados de la fuente y los del receptor: la información comunicacionalmente relevante del receptor depende de los estados de la fuente, y no a la inversa. Naturalmente, esta dependencia no es simétrica y es en estos términos en los cuales es posible establecer que la comunicación es un proceso asimétrico.

Algunas presentaciones más actuales de la teoría de Shannon introducen incluso esta característica en términos de un teorema: el teorema de la "desigualdad del procesamiento de datos" ("data processing inequality") (véase Cover y Thomas 1991, p. 32). Informalmente, la desigualdad establece que ninguna operación local sobre el destinatario puede crear información, sino que ésta depende exclusivamente de la fuente, es decir, la información no puede crearse ex nihilo.

La otra propiedad a destacar es que la comunicación es un proceso productivo o un hacedor de diferencias. La dependencia entre destinatario y fuente no sólo permite establecer una asimetría en la comunicación sino que también exhibe un vínculo más fuerte entre ambos sistemas. El proceso de comunicar una instancia de información es un proceso generador de diferencias, en la medida en que el acto de seleccionar un mensaje en la fuente es lo que produce una diferencia específica en los estados del receptor. Comunicar algo es precisamente producir esta diferencia.

Esta misma idea puede expresarse en términos contrafácticos. La idea de generar una diferencia se basa en establecer qué habría sucedido si cierta acción no hubiese tenido lugar. En una situación 
comunicacional, un cambio en los estados del receptor sólo pudo haber sido generado porque se llevó a cabo una acción en la fuente (en particular, la selección de un mensaje determinado, que luego fue codificado, comprimido y finalmente enviado): nada habría ocurrido si ningún mensaje hubiera sido seleccionado, codificado, comprimido y enviado.

\section{Interpretaciones del concepto de información comunicacional}

Hasta el momento hemos restringido considerablemente nuestro objeto de análisis. Partiendo de una noción sumamente general de la información, hemos arribado a una noción mucho más específica y concreta: información estadística comunicacional. Sin embargo, a pesar de estas precisiones, estamos aún lejos de obtener un concepto filosóficamente preciso. La información en contextos comunicacionales, ¿es de naturaleza epistémica o es alguna clase de entidad física? ¿Cuál es el referente del término "información”? ¿Es un concepto abstracto sin referencia, mero flatus vocis? Desafortunadamente, las teorías formales de la información no se pronuncian acerca de cuál es la naturaleza de la información ni permiten fijar una interpretación como privilegiada; por lo anterior, para obtener una respuesta a estas interrogantes resulta necesario dotar al concepto de una interpretación (Lombardi 2004).

\subsection{Interpretación epistémica}

De acuerdo con la interpretación epistémica, tal como sucede con la noción cotidiana, el concepto de información está fuertemente vinculado a la idea de conocimiento: cuando escuchamos las noticias, recibimos información acerca de ciertos sucesos que ignorábamos previamente; gracias a la información recibida, conocemos algo que previamente no conocíamos. Precisamente, esta interpretación intenta recoger estas intuiciones y brindarles un tratamiento sistemático que permita elucidar aquellas propiedades cognoscitivas y epistémicas de la información.

Un claro representante de esta interpretación es Fred Dretske, quien sostiene que si bien la teoría formal de Shannon (con modificaciones, véase Lombardi 2005) brinda la definición formal del concepto de información, no obstante, en lo que se refiere al contenido, la información se define por el conocimiento que produce. En palabras del autor, la información "es un producto (commodity) que, dado el recipiente adecuado, es capaz de producir conocimiento" (Dretske 1981, p. 47). Desde este punto de vista, la información está 
siempre vinculada a un incremento en el conocimiento de la persona que la recibe; en esta línea, Donald MacKay sostiene: "decimos que hemos ganado información cuando sabemos algo ahora que no sabíamos antes; cuando "lo que sabemos' cambió" (MacKay 1969, p. 10). La misma idea aparece incluso en libros de texto sobre teoría de Shannon: "la información se mide como la diferencia entre el estado de conocimiento del receptor antes y después de la comunicación de información" (Bell 1957, p. 7). A partir de esta interpretación, la información es considerada un ítem mental-epistémico relacionado con el incremento de conocimiento de quien la adquiere.

Es importante subrayar que, cuando asumimos este tipo de interpretación, la transmisión de información no requiere necesariamente una conexión física entre fuente y receptor. Sin embargo, las probabilidades que vinculan a ambos elementos no deben ser meras correlaciones accidentales. Efectivamente, cuando una correlación entre dos variables es meramente accidental, el valor que adopta una de ellas nada nos dice acerca del valor de la otra. Por lo tanto, conforme a esta interpretación, las probabilidades involucradas en el contexto comunicacional no son correlaciones de facto, sino que deben ser manifestaciones de regularidades legaliformes subyacentes.

\subsection{Interpretación física}

Por su parte, la interpretación física forma parte de las intuiciones cotidianas de los físicos e ingenieros en comunicación que diariamente lidian con problemas prácticos o teóricos donde la noción de información resulta central. De acuerdo con esta interpretación, el concepto de información no guarda ningún tipo de relación con el conocimiento, sino que se vincula con alguna magnitud o entidad física. Tradicionalmente, el principal interés de la ingeniería comunicacional ha sido optimizar la transmisión de información por medio de señales físicas, cuya energía y ancho de banda está restringido por limitaciones tecnológicas y económicas. Es destacable que la capacidad del canal se mide en bits por segundo: en el grado en que la unidad de medida del flujo de información está fuertemente vinculada con las magnitudes físicas tradicionales, la tentación de considerar la información como algún tipo de entidad o magnitud física será difícil de evitar.

En este sentido, la información es algo que se puede generar en un punto del espacio físico y transmitir a otro punto; que se puede acumular, almacenar y convertir de una forma a otra (Rovelli 1996). Uno de los exponentes que con más fuerza ha defendido la interpretación 
física de la información es Rolf Landauer, quien sostiene: "la información no es una entidad abstracta incorpórea; está siempre ligada a una representación física. Se representa grabando sobre una tabla de piedra, un espín, una carga, un agujero en una tarjeta, una marca sobre un papel o sobre alguna otra cosa equivalente" (1996, p. 88).

Uno de los aspectos centrales de la interpretación física de la información es su vínculo con un conocido dictum: "no hay información sin representación". Esto indica que la transmisión de información entre dos puntos del espacio físico requiere una señal portadora, es decir, un proceso físico que se propague de un punto a otro del espacio físico. En virtud de la naturaleza física de la información, su dinámica se rige a su vez por las leyes de la física (Landauer 1991, p. 29; véase también Bennet y Landauer 1985).

Una manera usual de entender la interpretación física es señalando las analogías entre el concepto de información y el de energía. Algunos autores, por ejemplo, han considerado que la información tiene su mismo estatus ontológico (Stonier 1996): si bien la energía no es considerada algo material en sentido de un ítem concreto, sí es algo que puede ser generado, acumulado, almacenado, procesado y convertido de una forma a otra, y transmitido de un punto del espacio a otro, por ser algo evidentemente físico.

\subsection{Interpretación deflacionista}

En los últimos años ha surgido una nueva interpretación de la información que pretende disolver todas las problemáticas y callejones sin salida en los que las anteriores interpretaciones han caído (tal como veremos en la sección siguiente). Defendida fundamentalmente por Christopher Timpson, esta interpretación deflacionista argumenta que la "información" es meramente un término de masa abstracto que no tiene referencia (2004, p. 7).

Luego de determinar un sentido técnico de la información, y de presentar la teoría de Shannon, Timpson distingue entre "bits" y "piezas" de información. Esta distinción pretende mostrar, por diferentes medios, la tesis central de la propuesta filosófica de Timpson: la información es una entidad abstracta, qua cantidad (bits) y qua pieza de información.

Por un lado, resulta obvio que la información qua cantidad es una entidad abstracta tal como lo son las entidades matemáticas que miden cierta magnitud. Hablar de información qua cantidad es meramente hablar acerca de la capacidad de comprensión máxima de los mensajes que produce una fuente de información y de sus propieda- 
des estadísticas (Timpson 2013, p. 22). De acuerdo con Timpson, no hay ningún misterio filosófico detrás de esta noción de información.

En cambio, cuando queremos hablar de información qua pieza, la cuestión se torna filosóficamente más sustantiva. El carácter abstracto de la información en cuanto pieza de información recae en la distinción metafísica entre tipos (types) y casos (tokens). En términos generales, esta distinción remite a la diferencia entre una clase general de cosas y sus instancias concretas, aunque los límites no sean siempre claros (véase Wetzel 2009). En el caso particular de Timpson, la inclusión de esta distinción en la discusión sobre el concepto de información busca señalar la diferencia que existe entre "los sistemas concretos que emite una fuente y el tipo que esas emisiones instancian" (Timpson 2004, p. 22). Si información es aquello producido por una fuente de información y que será reproducido en el destinatario, lo que realmente interesa desde el punto de vista comunicacional es reproducir la secuencia-tipo generada en la fuente mediante otras instancias-casos del mismo tipo. Por ello, según Timpson:

La producción de una secuencia-caso y luego la consecuente producción de otro caso del mismo tipo (o al menos la posibilidad de tal consecuente producción) es lo que constituye la transmisión de la secuencia-tipo. La secuencia-tipo es lo que se transmite mediante ese par de producciones de secuencia-caso; la secuencia-tipo es la pieza de información que la fuente produce. (Timpson 2013, p. 24)

Dado que los tipos son entidades abstractas, y que una pieza de información es una secuencia-tipo, el autor infiere que las piezas de información también son entidades abstractas.

El carácter abstracto de la información conduce a Timpson a generalizar el proceso comunicativo en términos de reproducción de patrones o estructuras. En sus palabras: "la idea básica es la de un patrón o una estructura: algo que puede realizarse repetidamente en diferentes instancias y, tal vez, por diferentes medios" (Timpson 2013 , p. 18). Desde este punto de vista deflacionista, el proceso de comunicar se reduce a reproducir en el destinatario el mismo patrón generado en la fuente. En otras palabras, comunicar es mapear una estructura generada en un lugar en otro: es un isomorfismo entre estructuras (Timpson, comunicación personal).

\section{Dificultades de las interpretaciones}

Cada interpretación conduce a una comprensión diferente de la naturaleza de la información: si observamos la teoría de Shannon bajo 
la lente de la interpretación física, consideraremos que el término "información" refiere a un tipo de entidad que constituye nuestro mundo, al igual que la energía. Por el contrario, si asumimos una interpretación deflacionista de la información, supondremos que de lo que habla esa teoría es meramente de un concepto abstracto sin referencia en el mundo real: la teoría de Shannon no describe cómo se comporta o cuáles son las propiedades de una entidad que está en el mundo.

Hay un sentido más interesante, que no se ha considerado lo suficiente, en el cual las interpretaciones constriñen la visión del concepto de información en situaciones comunicacionales. Dependiendo de qué interpretación asumamos, ciertas situaciones que involucran algún tipo de transmisión de información podrían incluso no ser consideradas como efectivamente comunicacionales. Es decir, no todas las interpretaciones conciben las mismas situaciones como comunicacionales. Por lo tanto, más allá de los problemas filosóficos particulares que cada interpretación pueda tener, cada una de ellas tiene el desafío de capturar como comunicacionales o informacionales aquellas situaciones que queremos capturar como tales.

El problema central es que ninguna de las interpretaciones presentadas logra este cometido con éxito: mientras que algunas pueden conducir a aceptar como informacionalmente relevantes situaciones que a todas luces no lo son, otras dejan de lado situaciones donde se admitiría que existe comunicación. En otras palabras, cada interpretación resulta adecuada en ciertos casos, pero falla rápidamente en otros. Ninguna parece ser lo suficientemente efectiva como para ofrecernos un enfoque completo e integral de qué es y cómo se usa la información comunicacional en el campo de la filosofía de la física.

4. 1. Problemas de la interpretación epistémica y deflacionista: correlaciones accidentales y comunicación

La interpretación epistémica no sólo permite recoger de manera sistemática nuestras intuiciones cotidianas respecto de la naturaleza semántico-cognoscitiva de la información, sino que también resulta útil en diferentes ámbitos. Por ejemplo, se ha aplicado con éxito para conceptualizar científicamente las capacidades humanas de adquirir conocimiento (véase, por ejemplo, Hoel, Albantakis y Tononi 2013). También se ha apelado al concepto de información en su interpretación epistémica, en filosofía, para complementar la teoría formal de la información con una dimensión semántica (MacKay 1969, 
Nauta 1972, Dretske 1981). Sin embargo, los criterios que suministra la interpretación epistémica para identificar situaciones comunicacionales resultan demasiado laxos en ciertas situaciones. Esto conduce a aceptar como comunicacionales situaciones que no parecen serlo (véanse, por ejemplo, Lombardi, Fortin y Vanni 2015, Lombardi, Holik y Vanni 2016). Como veremos, buena parte de estos problemas se derivan de la manera en la que estas interpretaciones entienden el tipo de conexión existente entre fuente y receptor.

Supongamos una antena de transmisión $T$ que emite una señal electromagnética y dos televisores, $T V_{1}$ y $T V_{2}$, que reciben esta señal sintonizados en el mismo canal. Entre estos elementos podemos establecer algunos vínculos y correlaciones potenciales de manera clara: por un lado, existe una correlación entre los estados de los televisores y $T$, de modo que podemos saber qué sucede en cualquiera de los dos televisores (por ejemplo, qué película están pasando) si conocemos el estado de $T$. Por otro lado, es posible establecer también correlaciones entre los estados de los dos televisores: mirando el estado de $T V_{1}$ podemos conocer el estado de $T V_{2}$. La pregunta informacionalmente relevante es: ¿cuáles de estas correlaciones representan situaciones comunicacionales?

Comenzado con el caso más obvio, es evidente que existe genuina comunicación entre $T$ y los dos televisores. Pero, ¿qué sucede con las correlaciones entre los estados de los dos televisores? Este caso resulta un poco más problemático. Desde el punto de vista de la interpretación epistémica, deberíamos aceptar que, una vez que se conocen las correlaciones entre los dos televisores, hay transmisión de información ya que es posible aprender algo acerca de $T V_{1}$ con sólo mirar $T V_{2}$ y viceversa. En palabras del propio Dretske: "si las relaciones estadísticas que definen equivocidad y ruido entre $S$ y $R$ son apropiadas, entonces hay un canal entre esos dos puntos, y hay información entre ellos, aunque no haya un vínculo físico directo entre $S$ y $R$ " (Dretske 1981, p. 38).

Naturalmente, el mismo argumento valdría para la interpretación deflacionista en la medida en que existe la reproducción de un patrón de secuencias-tipo: si tomamos como fuente a $T V_{1}$ y como receptor a $T V_{2}$, ciertamente podríamos trazar un mapeo uno a uno entre los estados de ambos televisores y considerar la situación como genuinamente comunicacional. Sin embargo, es posible señalar un problema adicional para esta interpretación: si la existencia de una situación comunicacional depende exclusivamente de poder establecer correlaciones uno-a-uno entre los estados de la fuente y del receptor, entonces 
no es posible concebir como comunicacionales situaciones en las que haya ruido o equivocidad. Definicionalmente, una comunicación con ruido no es una situación en la que existan correlaciones uno-a-uno entre los estados de la fuente sino que, por el contrario, las correlaciones son uno-a-muchos (véase, por ejemplo, Cover y Thomas 1991, p. 7). Más aún, las situaciones reales de comunicación prácticamente siempre involucran algún grado de ruido o equivocidad; justamente, la ingeniería en comunicaciones busca reducir al mínimo estas cantidades distorsionantes.

A la luz de estos problemas, y dado que nuestro ámbito de discusión es la filosofía de la física, es razonable pensar que la interpretación física de la información es la que resulta más adecuada en estos contextos. Sin embargo, esta interpretación enfrenta serios problemas cuando nos movemos al ámbito de la mecánica cuántica, en particular, cuando consideramos el protocolo de teleportación cuántica de información.

\subsection{Problemas de la interpretación física: el protocolo de teleportación cuántica}

La interpretación física de la información concibe lo físico en térmicos clásicos, es decir, no cuánticos: los sistemas físicos se encuentran localizados en el espacio, y para que dos sistemas preserven las correlaciones entre sus propiedades deben interactuar a través de señales físicas que "viajan" a través del espacio invirtiendo en ello un tiempo no nulo. Es precisamente esta concepción de lo físico aquello que la mecánica cuántica pone en crisis, admitiendo la existencia de entidades físicas no localizadas y de correlaciones que se mantienen a través del enredamiento, a pesar de no encontrarse montadas sobre interacciones. La teleportación cuántica, posible gracias al enredamiento de sistemas cuánticos, pone en crisis la visión clásica de lo físico, al tiempo que se instituye como un fuerte obstáculo para la interpretación física tradicional de la información.

La implementación de protocolos de teleportación cuántica de información ha concentrado la atención de científicos y filósofos de la física y de la información en los últimos años. En esencia, el protocolo de teleportación cuántica consiste en que un estado cuántico desconocido $|\chi\rangle$ se transfiere de Alice a Bob con la ayuda de un par compartido de partículas preparadas en un estado enredado, más la asistencia de dos bits clásicos. El protocolo hace uso explícito de recursos eminentemente cuánticos (como el enredamiento de partículas) para transferir cierta cantidad de información haciendo uso de 
estados cuánticos (qubits). El fenómeno no es sólo sumamente atractivo, sino además impactante: al hacer uso del enredamiento cuántico de partículas, la comunicación podría llevarse a cabo sin que existiera un canal físico mediante el cual la información contenida en el estado cuántico pudiera transmitirse. Es precisamente esta peculiaridad lo que se constituye como un obstáculo difícilmente superable para la interpretación física (en la próxima sección volveremos sobre el tema, cuando analicemos el protocolo de teleportación desde el enfoque manipulabilista).

Con vistas a elucidar conceptualmente este caso, la pregunta central es cómo ha obtenido Bob la información. En principio, no existe ningún canal cuántico ni ninguna señal que porte la información que Alice quiere transmitir a Bob: desde el punto de vista de la interpretación física, lo único que se transmite en sentido estricto son los dos bits enviados por el canal clásico; sin embargo, no es precisamente ésa la información que se quiere reproducir en el destinatario, sino aquella codificada por Alice en los estados cuánticos que son "teletransportados". En segundo lugar, cabe preguntarse en qué medida la teleportación es un genuino caso de comunicación desde el punto de vista de la interpretación física: si la ausencia de un canal físico y una señal portadora es lo que permitía a la interpretación física desechar por comunicacionales meras correlaciones sin señal portadora, ¿por qué deberíamos aceptar que la teleportación cuántica es un genuino caso de transmisión de información?

Vale la pena subrayar que buena parte de la repercusión obtenida por la interpretación deflacionista ha radicado en poder brindar una respuesta simple y convincente del fenómeno de teleportación: naturalmente, el problema se disuelve ni bien comprendemos que nada es realmente transmitido entre Alice y Bob, y que los misterios y problemas derivados de la teleportación se deben a una errónea adjudicación de referencialidad a un término que no la tiene. Pero si bien asumir una interpretación deflacionista podría disolver el problema de la teleportación, nos enfrentaría a otro tipo de problemas, tal como los mencionados en la subsección anterior.

Algunos científicos y filósofos han propuesto salidas al problema de la teleportación desde una interpretación física; sin embargo, estas respuestas exigen compromisos filosóficos adicionales que pueden resultar controversiales y ser acusados de poseer un carácter exclusivamente ad hoc. Roger Penrose (1998) ha defendido que, para dar sentido a la teleportación cuántica y entender cómo Bob ha recibido la información, es necesario postular que la información viaja hacia 
el pasado (hasta el momento en que las dos partículas interactúan por primera vez) y luego hacia el futuro (cuando Bob mide sobre su partícula del par enredado). David Deutsch y Patrick Hayden (2000), por su parte, han argumentado que la información cuántica en realidad viaja oculta en los dos bits clásicos que Alice envía a Bob mediante un canal clásico físico convencional.

Si bien las respuestas de Penrose y de Deutsch y Hayden son sustancialmente diferentes, concuerdan en un punto: la interpretación física depende de poder determinar un canal físico por el cual se transmite la información, y las dos respuestas se comprometen con criterios extras para poder conservar esta idea medular de la interpretación. En este sentido, parece que quien defienda la interpretación física tradicional se halla en una especie de encrucijada de difícil resolución:

(a) Por un lado, si asume que el protocolo de teleportación es un genuino caso de comunicación, entonces está obligado a responder a la pregunta de cómo la información llega a Bob señalando cuál es el vínculo físico mediante el cual se transmite la información. Como vimos, esto conduce a respuestas como la de Penrose o Deutsch y Hayden.

(b) Por otro lado, dado que la interpretación es incapaz de señalar un canal físico entre fuente y receptor, la teleportación cuántica de información no sería un caso genuino de transmisión de información. Esta conclusión sería poco deseable dadas las exitosas aplicaciones del protocolo de teleportación en los últimos años.

En lo que resta del artículo ofreceremos una nueva interpretación físico-causal de la información, que intenta superar las dificultades mencionadas. En particular, pretendemos lograr una mejor integración entre el concepto de información y el contexto en el cual el concepto surge, el contexto comunicacional. Ciertas características del contexto comunicacional nos permitirán poner en claro cuáles son los procedimientos necesarios y los requisitos mínimos para transmitir información. En concreto, nuestra interpretación físico-causal buscará elucidar el concepto de información comunicacional en términos causales, apelando a las teorías manipulabilistas e intervencionistas de la causación desarrolladas en los últimos veinte años. A la luz de nuestro enfoque, una situación comunicacional exige una estructural causal donde la información es una propiedad física de los sistemas 
que podemos manipular: al intervenir en los estados de la fuente, podemos controlar de una manera específica los estados del receptor. Comunicar requiere esencialmente poder llevar a cabo este tipo de modificaciones controladas. En la siguiente sección presentaremos los lineamientos generales de las teorías manipulabilistas de la causación, focalizándonos en su versión intervencionista (Woodward 2003, Pearl 2000); dicha sección servirá de marco teórico para respaldar el aspecto causal de la interpretación que proponemos.

\section{Causación y manipulabilidad}

En una primera aproximación, se nos podría objetar que pretendemos aclarar un concepto oscuro - el de información - mediante un concepto más oscuro aún - el de causa-; sin embargo, nuestro propósito es mucho más modesto. Lo que buscamos es una teoría de la causación que nos ofrezca un criterio práctico y claro para identificar relaciones causales en diferentes situaciones. En particular, una teoría que nos permita diferenciar situaciones que presuponen una estructura causal subyacente de situaciones donde sólo hay meras correlaciones.

\subsection{El papel de la manipulabilidad}

Los problemas en torno a la causación y sus múltiples respuestas atraviesan toda la historia de la filosofía occidental. Mientras que algunos autores han considerado que la causación tiene un profundo vínculo conceptual con las condiciones semánticas y justificativas de las afirmaciones contrafácticas, tal como sostienen las teorías lewisianas de la causación (Lewis 1973), otros han sostenido que la causación es un fenómeno de naturaleza eminentemente física, fenómeno que puede ser entendido en términos de flujo de energía (Fair 1979), en términos de procesos físicos (Russell 1948) o como transferencia de propiedades (Ehring 1997), o como transferencia de una magnitud conservada (Dowe 2000). El célebre filósofo inglés Bertrand Russell (1912) incluso defendió una posición escéptica respecto de la causación, abogando por la completa exclusión de la palabra "causa" del vocabulario filosófico y científico.

Más allá de la enorme variedad de teorías y enfoques acerca de la causación, no existe consenso alguno entre filósofos acerca de qué es una relación causal o de cómo justificarla. Peor aún, como afirma James Woodward, tales enfoques han tenido "un impacto relativamente pequeño en la filosofía de la ciencia y la ciencia en general" (2003, p. 4). Sin embargo, resulta evidente que, tanto en nuestra 
vida cotidiana como en el trabajo científico habitual, somos capaces de seguir criterios intuitivos que nos permiten distinguir causas de meras correlaciones. Precisamente, uno de los rasgos característicos que usualmente identificamos a la hora de establecer esta clase de distinciones es la capacidad de control y manipulación que una relación causal involucra. Esta idea fue precisada y definida por Nancy Cartwright (1979) en términos de estrategias efectivas e inefectivas: una estrategia efectiva es posible sólo si existe una relación causal entre estados o variables de distintos sistemas, pues es la que permite el control de un estado o variable de un sistema mediante la manipulación de algún estado o variable de otro sistema. Las teorías manipulabilistas de la causación pretenden, precisamente, capturar y sistematizar estos criterios e intuiciones. La idea central consiste en que sólo las relaciones causales, y no las meras correlaciones, pueden ser utilizadas por nosotros con vistas a obtener algún tipo de resultado particular (Frisch 2014).

Las primeras versiones manipulabilistas han buscado reducir los términos causales a términos no causales, como el concepto de "libre agencia" (von Wright 1971, Price 1991, Menzies y Price 1993). En esta temprana teoría manipulabilista, se puso el acento en las capacidades de manipulación que posee un agente, al menos en sentido hipotético: "un suceso $A$ es una causa de un suceso distinto $B$ sólo en caso de que producir la aparición de $A$ sea un medio efectivo por el cual un agente libre podría producir la aparición de $B$ " (Menzies y Price 1993, p. 187). No obstante, estas primeras versiones fueron objeto de numerosas críticas y resultaron rápidamente desacreditadas: su sesgo fuertemente antropocéntrico (la necesidad de un agente humano que pueda llevar a cabo la manipulación) y su carácter circular (reducir el concepto de causa a nociones que también son causales, como "hacer" o "producir") figuran entre las principales objeciones a esta versión del manipulabilidad (véanse Woodward 2016, Frisch 2014).

Buscando evitar estas críticas, una nueva versión del manipulabilismo apareció en la escena filosófica: la versión intervencionista desarrollada principalmente por James Woodward y Judea Pearl. Renunciando a las aspiraciones reduccionistas usuales y ofreciendo en su lugar criterios sistemáticos para identificar relaciones causales, esta teoría se ha vuelto particularmente popular entre científicos sociales y público no filosófico. El espíritu de este enfoque puede resumirse en las siguientes palabras de Mathias Frisch: "los resultados de intervenciones en un sistema son guías para exhibir la estructura causal 
del sistema" (Frisch 2014, p. 78). En esta versión basaremos nuestra interpretación físico-causal de la información.

\subsection{Causación e intervención}

¿Qué es una relación causal? ¿Qué significa " $C$ causa $E$ ”? De acuerdo con el espíritu del manipulabilismo, " $C$ causa $E$ " significa que podemos manipular $E$ (el efecto) manipulando $C$ (la causa). Un efecto causal se define a partir del cambio o de la diferencia generada en $E$ a partir de un cambio o diferencia ocasionada en $C$; y es esto precisamente lo que nos permite el control o la manipulación de $E$. Citando a Woodward:

La afirmación de que $C$ causa $E$ significa que hay una posible manipulación de algún valor en $C$, la cual, dada las condiciones apropiadas (tal vez, incluyendo que otras variables permanezcan fijas durante la manipulación), cambiará el valor de $E$ o la distribución de probabilidad de $E$. (Woodward 2003, p. 40)

La versión intervencionista se articula en torno a dos elementos centrales: por un lado, la relación causal es una relación entre variables; por otro lado, la idea de "cambiar un valor" se representa mediante la noción de intervención. El primer elemento describe cuál es el objeto de manipulación y cómo los cambios en el valor de una o más variables alteran el valor de otras variables (Woodward 2003, p. 39); mientras que la noción de intervención permite capturar, en un lenguaje no antropocéntrico, la idea de manipular el valor de $C$ para determinar si $C$ causa $E$ por medio de una manipulación experimental factual o ideal. De esta manera, que $C$ sea una causa de $E$ significa que "sólo bajo una intervención que cambie el valor de $C$ (sin que ninguna otra intervención ocurra), hay un cambio asociado en el valor de $E^{\prime \prime}$ (Woodward 2007, p. 73).

En términos más precisos, una intervención $(I)$ es un proceso causal que permite poner al descubierto la relación causal $X \rightarrow Y$ bajo investigación. Se trata de un proceso causal exógeno respecto de la relación causal que se pretende identificar; Sin embargo, no cualquier acción exógena sobre un sistema cuenta como una intervención genuina: deben satisfacerse ciertas condiciones. Woodward (2003, p. 98) establece las siguientes cuatro condiciones para una intervención genuina:

(i) $I$ causa $X$. 
(ii) $I$ actúa como un interruptor para todas las otras variables que causan $X$; esto es, ciertos valores de $I$ son tales que, cuando $I$ adopta esos valores, $X$ deja de depender de los valores de otras variables y pasa a depender sólo de los valores que adopta $I$.

(iii) Cualquier camino que va de $I$ a $Y$ pasa a través de $X$. Es decir, $I$ no actúa causalmente de manera directa sobre $Y$, y no es causa de cualquier otra causa de $Y$ que sea distinta de $X$ excepto, por supuesto, aquellas causas que causan $Y$ y que se encuentran en la misma conexión $I-X-Y$; esto es, excepto para (a) cualquier causa de $Y$ que sea efecto de $X$ (i.e., una variable causal que está entre $X$ e $Y$ ) y (b) cualquier causa de $Y$ que está entre $I$ y $X$ y no tiene efecto sobre $Y$ de manera independiente de $X$.

(iv) $I$ es (estadísticamente) independiente de cualquier variable $Z$ que causa $Y$ y que está en un camino directo que no pasa a través de $X$.

\section{Transmisión de información y estructura causal: no hay información sin manipulación}

Resumamos el camino recorrido. Luego de presentar las diferentes interpretaciones del concepto de información más extendidas en filosofía de la física, expusimos algunos de sus problemas y dificultades. Allí subrayamos que, si bien estas interpretaciones resultan útiles en situaciones específicas, ninguna de ellas es suficiente para brindar un abordaje más completo e integral del concepto de información en contextos comunicacionales. La interpretación físico-causal que esbozaremos en esta sección pretende no sólo superar las dificultades del resto de las interpretaciones, sino también brindar las condiciones y los requisitos prácticos para caracterizar e identificar situaciones comunicacionales.

La interpretación que queremos defender se apoya en dos pilares: (a) por un lado, entiende la propiedad de "ser físico" en términos no sustanciales, esto es, "físico" no implica "entidad material"; (b) por otro lado, incorpora el concepto de causación y manipulabilidad a los conceptos de comunicación y de información. Si bien nuestro foco de interés estará puesto en el segundo pilar, en la medida en que es el más innovador y el que permitirá articular los diferentes conceptos involucrados, dedicaremos algo de espacio a precisar en qué sentido nuestra interpretación podría estar enmarcada dentro de las interpretaciones físicas. Sin embargo, vale la pena insistir en que 
hay cierta independencia entre estos dos pilares: todo lo desarrollado respecto del punto (b) también podría ser utilizado para apuntalar, por ejemplo, una interpretación deflacionista de la información. La razón de por qué no hemos seguido esta vía en este trabajo radica en ciertos problemas conceptuales de la interpretación deflacionista (véase, por ejemplo, Lombardi, Fortin y López 2016); en cambio, creemos que una interpretación física modificada no sólo está libre de tales problemas, sino que también encaja de una manera más natural en el ámbito de la filosofía de la física.

Las interpretaciones físicas de la información han entendido, usualmente, la noción de "físico" como si refiriera a algún tipo de entidad, flujo material o sustancia espacio-temporalmente localizada; precisamente, las críticas de Timpson a la interpretación física presuponen esta noción sustancialista de la información (véase Timpson 2013, cap. 1). Nuestra interpretación físico-causal no es una interpretación física en ese sentido. Si por el término "físico" entendemos algún tipo de referente concreto y espacio-temporal, entonces concordamos plenamente con la posición deflacionista de Timpson: la información no es ningún tipo de sustancia concreta que fluya en el mundo. No obstante, Timpson entiende por "físico" sólo entidades concretas espacio-temporales. Creemos que puede hacerse una distinción más sutil.

Nuestra interpretación incluye en el ámbito de lo físico no sólo entidades concretas espacio-temporales, sino también ciertas propiedades que caracterizan a este tipo de entidades. Y si bien la naturaleza metafísica de las propiedades es abstracta (como generalmente se acepta), eso no implica que no podamos distinguir entre propiedades predicables de entidades físicas (como masa, energía o espín) de propiedades no físicas (por ejemplo, "ser impar" o "ser una tautología"). La distinción permite establecer el rango ontológico adecuado para el concepto de información comunicacional: la información es física en cuanto es una propiedad que tienen ciertos sistemas físicos (fuentes y receptores), señales y portadores de información, pero que no tienen otros tipos de entidades (al menos en términos comunicacionales y conforme a las teorías formales que hemos presentado): números o enunciados no "portan" información en este mismo sentido. Además, al calificar la información comunicacional como física, establecemos que es una propiedad que forma parte principalmente del vocabulario científico de las ciencias físicas.

El segundo pilar sobre el que se sostiene la interpretación físicocausal consiste en integrar causación y manipulabilidad al ámbito de 
la información comunicacional. En términos generales, la idea es que la comunicación (es decir, todas las instancias donde hay transferencia de información) descansa sobre una estructura causal que podemos poner al descubierto utilizando criterios manipulabilistas e intervencionistas. En esencia, el corazón de nuestro argumento consiste en mostrar que la existencia misma de situaciones comunicacionales depende de poder llevar a cabo ciertas modificaciones en los estados de la fuente con el propósito de obtener ciertas modificaciones en los estados del receptor, estableciendo correlaciones causalmente relevantes entre esos estados. En otras palabras, los estados del destinatario deben poder ser manipulados de manera controlada al intervenir en los estados de la fuente.

En consonancia con estas ideas, si existen correlaciones entre estados de dos sistemas físicos, podremos decir que hay transferencia de información entre los sistemas sólo si somos capaces de controlar los estados de uno de ellos al intervenir sobre los estados del otro. En este sentido, las situaciones comunicacionales parecen satisfacer un principio del siguiente tipo:

Principio de manipulabilidad (PM). Para que exista una situación comunicacional, es necesario que (i) sea posible identificar los estados del sistema-fuente mediante los estados del sistemadestinatario y (ii) la correlación entre los estados de los dos sistemas refleje un vínculo causal genuino, evidenciable mediante criterios manipulabilistas.

Veamos cómo funciona nuestra interpretación en un caso concreto. Recordemos el ejemplo que mencionamos en la sección 5: una antena transmisora y dos televisores cuyos estados están perfectamente correlacionados. La discusión de este ejemplo giraba en torno a qué correlaciones resultaban comunicacionalmente relevantes de acuerdo con cada interpretación. Desde nuestro enfoque, el punto central para dirimir si ciertas correlaciones entre estados de sistemas físicos implican situaciones comunicacionales consiste en mostrar que existe una estructura causal subyacente. Como primer paso, necesitamos aislar los sistemas correlacionados y evaluar si existe una relación causal entre ellos. Comencemos con el caso más sencillo: la antena transmisora $T$ y los dos televisores que reciben señales de la antena televisora. La antena transmisora puede estar en dos estados: $T=1$ (cuando está encendida) o $T=0$ cuando está apagada; de la misma manera, los televisores pueden estar en dos estados posibles, $T V_{i}=1$ 
(cuando sintonizan una señal determinada) o $T V_{i}=0$ (cuando no sintonizan ninguna señal).

Para evaluar si existe un vínculo causal entre $T$ y los dos televisores, y por ende transmisión de información, debemos definir una intervención posible sobre la alegada causa (fuente): supongamos que llevamos a cabo una intervención " $d o$ " sobre $T$ que cambia el valor de $T=1$ a $T=0$. La existencia de una relación causal entre la antena (la causa-fuente) y los estados de los televisores (efectos-receptores) queda en evidencia cuando, al intervenir sobre el estado de la antena $(d o(T))$, el estado de los televisores también se ve alterado; o, en términos más precisos, cuando podemos manipular los estados de los televisores al intervenir sobre algún estado de la antena. En este caso concreto, resulta evidente que al intervenir sobre el estado de $T$, la señal se interrumpe y se modifican los estados de los televisores cumpliendo la siguiente desigualdad:

$$
\operatorname{Prob}\left(T V_{i}=0\right) / d o(T) \neq \operatorname{Prob}\left(T V_{i}=0\right)
$$

Es decir, la probabilidad de que los televisores se encuentren en el estado apagado dada la intervención que desactiva la antena es distinta de la probabilidad de los televisores se encuentren en el estado apagado sin llevar a cabo ninguna intervención sobre la antena. Esta desigualdad es lo que sugiere que existe una relación causal entre $T$ y $T V_{1}-T V_{2}$. La existencia de esta relación causal es lo que nos habilita, a partir de nuestro enfoque, a hablar de transferencia de información entre $T$ y los dos televisores. Dado que la situación satisface PM, se trata de una situación comunicacional.

Consideremos ahora la otra correlación, comunicacionalmente mucho menos clara que la anterior: la que existe entre los dos televisores. Como señalamos antes, no suele suponerse que nos comunicamos a través de televisores sintonizados en la misma estación: nada puede hacerse en un televisor para enviar información que pueda recibirse en el otro. En efecto, las correlaciones entre los dos televisores se deben a alguna clase de causa común. Sin embargo, ni la interpretación epistémica ni la interpretación deflacionista podían recoger de una manera natural esta idea natural: según sus criterios para seleccionar situaciones comunicacionales, no había motivos claros y sólidos para considerar esta situación como no comunicacional. ¿Cómo abordar este caso particular desde el enfoque físico-causal?

Según nuestra interpretación, la estrategia para extraer una conclusión resulta sencilla: ninguna intervención sobre algún estado de $T V_{2}$ puede ocasionar algún cambio o modificación en $T V_{1}$. No parece 
haber ningún sentido según el cual podemos manipular $T V_{1}$ alterando alguna variable de $T V_{2}$. Pero si no es posible llevar a cabo esta manipulación, entonces no tenemos motivos para pensar que existe una relación causal subyacente $y$, sin una relación causal subyacente, no es posible determinar que existe transmisión de información entre los dos televisores. Conforme a nuestra interpretación, fácilmente podemos descartar correlaciones no causales como sustrato de genuinas situaciones comunicacionales, en la medida en que en tales correlaciones no se satisface PM.

La interpretación física tradicional también podía arribar a la misma conclusión utilizando como criterio la existencia de un canal físico y una señal portadora entre fuente y destinatario. Por lo tanto, ¿cuál es la ventaja de nuestra interpretación? La respuesta se halla cuando analizamos casos no tradicionales, como el del protocolo de teleportación cuántica. Como vimos, el caso resultaba un hueso duro de roer para la interpretación física, ya que parecía existir una situación comunicacional sin un canal físico o una señal portadora entre Alice y Bob. Analicemos ahora esta situación ateniéndonos a nuestra interpretación.

Especifiquemos con un poco más de detalle en qué consiste el protocolo de teleportación: Alice quiere enviar un mensaje a Bob utilizando (a) un par de partículas enredadas, y (b) un canal clásico por el cual se envían dos bits de información. Como primer paso para lograr el protocolo, Alice y Bob enredan el par de partículas cuánticas, tomando cada uno de ellos una partícula del par y separándose luego. La información que Alice quiere enviar a Bob se prepara y codifica en un estado cuántico $|\chi\rangle$. Luego, mediante un canal clásico, Alice envía dos bits de información clásica a Bob, quien, sobre esta base, decide el tipo de operaciones que debe llevar a cabo sobre su partícula del par enredado para obtener el estado que Alice preparó en su partícula del par. Gracias a la propiedad cuántica de enredamiento, cualquier operación local llevada a cabo sobre una de las partículas afectará de manera no local el estado de la otra partícula del par. Si Bob recibe los bits de información con estas instrucciones y mide en su partícula de la manera adecuada, "recibirá" el estado cuántico $|\chi\rangle$ que Alice preparó, el cual a su vez se destruye en la partícula de Alice.

La peculiaridad del protocolo de teleportación es que hace uso de propiedades de los sistemas cuánticos (como la propiedad de poder enredar sus estados) combinadas con recursos comunicacionales clásicos. Desde nuestra perspectiva, sin embargo, no es relevante qué marcos teóricos intervienen en la situación comunicacional. Por el 
contrario, para entender y analizar la situación comunicacional de manera completa, sólo resulta necesario especificar todas sus partes constituyentes, y luego aplicar los criterios manipulabilistas a cada una de ellas. En referencia al primer punto, el protocolo de teleportación consiste en: (a) dos partículas cuánticas enredadas, (b) ciertas operaciones locales que Alice lleva a cabo sobre una de las partículas del par enredado, y (c) dos bits de información que Alice envía a Bob mediante un canal clásico. El éxito del protocolo de teleportación depende de que Bob pueda obtener el estado que Alice transmitió, y este cometido depende de que estos elementos se articulen de la manera adecuada. A la luz de nuestra interpretación, el éxito comunicacional del protocolo depende de poder establecer las relaciones causales adecuadas entre estos elementos.

Evaluemos la situación a partir de los criterios manipulabilistas ya mencionados. Consideremos primero los dos bits que Alice envía a Bob con instrucciones para medir sobre su par y obtener el estado cuántico teletransportado. Considerando a Alice como la fuentecausa, ella puede encontrarse en tres estados posibles: $A=2$ cuando envía dos bits de información, $A=1$ cuando envía un bit de información, y $A=0$ cuando no envía información clásica; los estados de Bob (el efecto-receptor) serán, $B=2$ cuando Bob recibe dos bits de información, $B=1$ cuando recibe un solo bit de información, y $B=0$ cuando no recibe información clásica. Definamos ahora una intervención sobre Alice mediante la cual en lugar de enviar dos bits de información envía sólo uno. Tal como se ha explicado, de este modo el protocolo ya no tiene éxito: al llevar a cabo esta intervención, la instrucción necesaria para que Bob recupere el estado cuántico que Alice preparó sobre su partícula del par estará incompleta, e imposibilitará que Bob reconstruya el estado. El protocolo de teleportación fracasa en este caso, ya que Bob no puede obtener la información "cuántica" que Alice codificó y envío en el estado $|\chi\rangle$. El fracaso del protocolo al intervenir sobre la alegada causa de la manera indicada pone de relieve que existe una relación causal genuina entre enviar-obtener dos bits de información y poder reconstruir el estado cuántico. La teleportación perdería todo sentido comunicativo e informacional sin el envío/obtención de dos bits de información "clásica": podemos manipular el acceso de Bob al estado cuántico que Alice preparó controlando cuántos bits de información clásica envía Alice mediante un canal clásico.

Analicemos ahora los otros dos elementos del protocolo de teleportación. Supongamos que Bob recibe efectivamente dos bits de información clásica (en términos de la teoría intervencionista, ese 
mecanismo causal queda intacto y permanece aislado de cualquier intervención sobre Alice). Pero ahora definimos dos estados de Alice del siguiente modo: $A=|\chi\rangle$ y $A=|\phi\rangle$, indicando cuál es el estado cuántico que Alice preparará para enviar a Bob. Análogamente, definimos los estados de Bob como: $B=|\chi\rangle$ y $B=|\phi\rangle$, según qué estado reciba. En el caso en que $A=|\chi\rangle$, puede asegurarse que $B=|\chi\rangle$ y, por lo tanto, $\operatorname{Prob}(B=|\chi\rangle)=1$. Supongamos ahora que intervenimos sobre Alice para que prepare el estado $|\phi\rangle$, en lugar del estado $|\chi\rangle$, llevando a cabo la transformación $d o(A): A=|\chi\rangle \rightarrow A=|\phi\rangle$. ¿Qué estado obtendrá Bob, una vez que mida sobre su partícula enredada con la de Alice? Evidentemente, obtendrá el estado $|\phi\rangle$. Puesto que el estado $|\phi\rangle$ es cualquier estado, esto muestra que Bob no podrá obtener el estado $|\chi\rangle$ si Alice no prepara su partícula del par en ese estado: $\operatorname{Prob}(B=|\chi\rangle) / d o(A)=0$. En consecuencia, análogamente a lo que sucedía en el caso de los televisores y la antena transmisora (véase la ecuación 2), aquí se cumple:

$$
\operatorname{Prob}(B=|\chi\rangle) / d o(A) \neq \operatorname{Prob}(B=|\chi\rangle)
$$

Nuevamente, estos resultados muestran la existencia de una estructura causal subyacente entre Alice y Bob, independientemente de cualquier vínculo físico: el estado es transmitido de Alice a Bob, y lo que Bob recibe depende de lo que Alice haya preparado. Mediante qué mecanismo físico se envía la información cuántica, o qué tipo de relación causal es la que vincula a Alice con Bob, es algo que yace más allá de las pretensiones del enfoque manipulabilista de la causación y de nuestra interpretación físico-causal. Probablemente éste sea un problema de análisis filosófico de los fundamentos de la mecánica cuántica y, en particular, del enredamiento de partículas cuánticas.

El punto por resaltar aquí es que, si se aceptan los criterios manipulabilistas para identificar relaciones causales, debemos aceptar que el protocolo de teleportación se sustenta en una estructura genuinamente causal, independientemente de cuál sea su naturaleza o de cuán misterioso pueda parecer. Vale la pena insistir en esta cuestión: el propósito de una interpretación del concepto de información no tiene por qué elucidar los problemas filosóficos u ontológicos de la teoría que explica el comportamiento de los sistemas físicos utilizados para implementar el protocolo de comunicación; la naturaleza del enredamiento cuántico o el problema de la no localidad son preocupaciones de la filosofía de la mecánica cuántica, no de una interpretación del concepto de información comunicacional; lo que 
ella debe proveer son criterios claros que permitan especificar qué procedimientos prácticos son necesarios para transmitir información.

\section{Conclusiones}

En este artículo presentamos una interpretación físico-causal del concepto de información en contextos comunicacionales. Circunscribiendo nuestra discusión al ámbito de la filosofía de la física, primero ofrecimos un análisis pormenorizado del amplio abanico de enfoques y contextos que actualmente constituyen el campo de la filosofía de la información, lo cual nos posibilitó determinar con precisión en qué consiste el concepto técnico-estadístico de información. A su vez, señalamos que este concepto particular es el que se suele asumir y utilizar en contextos comunicacionales, contextos donde típicamente se han desarrollado teorías formales como la de Shannon.

Luego de este análisis, presentamos diferentes interpretaciones del concepto de información comunicacional: la interpretación epistémica, la interpretación física y la interpretación deflacionista. Al analizar estas interpretaciones, mostramos cómo cada una ellas cae en dificultades a la hora de identificar situaciones comunicacionales genuinas: mientras que la interpretación deflacionista y la interpretación epistémica pueden asumir como situaciones comunicacionales meras correlaciones entre estados de sistemas físicos, la interpretación física es incapaz de reconocer ciertos protocolos utilizados para transmitir información como genuinamente comunicacionales. Nuestra interpretación se planteó en este punto como una alternativa superadora de las interpretaciones tradicionales en el campo de la filosofía de la física y la información: asumiendo que la información es una propiedad física de sistemas físicos y que toda situación comunicacional debe satisfacer el llamado principio de manipulabilidad, mostramos cómo todo proceso de transferencia de información supone una estructura causal que es posible poner al descubierto mediante criterios manipulabilistas.

Es importante resaltar que la presente interpretación no introduce una equivalencia entre información comunicacional y causación manipulabilista. Desde nuestra perspectiva, la existencia de un vínculo causal es condición necesaria para que haya transmisión de información: no puede darse el caso de comunicación (transmisión de información) sin vínculo causal. Sin embargo, no descartamos la posibilidad de que existan vínculos causales no informacionales, en particular en los casos de causación natural no mediada por la acción humana, que el enfoque de Woodward conceptualiza con recursos 
contrafácticos (por ejemplo, la acción de la luna sobre las mareas terrestres, la dependencia de terremotos y erupciones volcánicas respecto de los movimientos tectónicos).

Creemos que, más allá de las ventajas para superar las dificultades de las otras interpretaciones, nuestra interpretación físico-causal permite obtener un concepto de información comunicacional más robusto y mejor articulado con el contexto en el que el concepto suele implementarse. Para ello, en lugar de elaborar una interpretación que sea fundamentalmente acerca de la naturaleza de la información, construimos nuestro enfoque con vistas a precisar qué procedimientos son necesarios para lograr transmitir información. Esto también permite que la interpretación se pueda extender a otros ámbitos, más allá de la filosofía de la física: el concepto de información está sumamente presente en la filosofía de la biología y la filosofía de las ciencias cognitivas, por ejemplo; y creemos que, con las modificaciones pertinentes, nuestra interpretación podría rendir sus frutos también allí.

En definitiva, nuestra interpretación físico-causal podría resumirse en el siguiente lema: no hay información sin manipulación. ${ }^{1}$

\section{BIBLIOGRAFÍA}

Bar-Hillel, Yehoshua, 1964, Language and Information: Selected Essays on Their Theory and Application, Addison-Wesley, Reading, Mass.

Bar-Hillel, Yehoshua y Rudolf Carnap, 1953, "Semantic Information", The British Journal for the Philosophy of Science, vol. 4, no. 14, pp. 147157.

Bell, D., 1957, Information Theory and its Engineering Applications, Pitman and Sons, Londres.

Bennett, Charles y Rolf Landauer, 1985, "The Fundamental Physical Limits of Computation", Scientific American, vol. 253, no. 1, pp. 48-56.

Cartwright, Nancy, 1979, "Causal Laws and Effective Strategies", Nô̂s, vol. 13, no. 4, pp. 419-437.

${ }^{1}$ Los autores agradecen sus fuentes de financiamiento a: John Templeton Foundation, Consejo Nacional de Investigaciones Científicas y Tecnológicas de la Argentina (CONICET) y Agencia Nacional de Promoción Científica y Tecnológica de la Argentina (ANPCyT). También se agradece al Grupo de Filosofía de las Ciencias de la Universidad Nacional de Buenos Aires, a los participantes del International Workshop: "What Is Quantum Information?" (Buenos Aires, 2015) por comentarios y observaciones a una primera versión de este artículo. Finalmente, agradecemos a los dos árbitros anónimos por contribuir a lograr una mejor versión del artículo. 
Chaitin, Gregory, 1966, "On the Length of Programs for Computing Binary Sequences", Journal of the Association for Computing Machinery, vol. 13, no. 4, pp. 547-569.

Cook, Thomas y Donald Campbell, 1979, Quasi-Experimentation: Design and Analysis Issues for Field Settings, Houghton Miflin, Boston.

Cover, Thomas y Joy Thomas, 1991, Elements of Information Theory, John Wiley and Sons, Nueva York

Deutsch, David y Patrick Hayden, 2000, "Information Flow in Entangled Quantum Systems", Proceedings of the Royal Society of London A, vol. 456, no. 1999, pp. 1759-1774.

Dowe, Phil, 2000, Physical Causation, Cambridge University Press, Nueva York.

Dretske, Fred, 1981, Knowledge and the Flow of Information, Basil Blackwell, Oxford.

Ehring, Douglas, 1986, "The Transference Theory of Causality", Synthese, vol. 67, no. 2, pp. 249-258.

Fair, David, 1979, "Causation and the Flow of Energy", Erkenntnis, vol. 14, no. 3, pp. 219-250.

Floridi, Luciano, 2017, "Semantic Conceptions of Information", The Stanford Encyclopedia of Philosophy (edición Primavera de 2017), Edward N. Zalta (ed.); disponible en: <http://plato.stanford.edu/archives/ spr2017/entries/information-semantic/> [fecha de consulta: 05/02/2017].

- 2011, The Philosophy of Information, Oxford University Press, Oxford.

Frisch, Mathias, 2014, Causal Reasoning in Physics, Cambridge University Press, Cambridge.

Hoel, Erik, Larissa Albantakis y Giulio Tononi, 2013, "Quantifying Causal Emergence Shows that Macro Can Beat Micro", Proceedings of the National Academy of Sciences, vol. 110, no. 49, pp. 19790-19795.

Kolmogorov, Andréi, 1965, "Three Approaches to the Quantitative Definition of Information", Problems of Information Transmission, vol. 1, no. 1, pp. 4-7.

Landauer, Rolf, 1996, "The Physical Nature of Information", Physics Letters A, vol. 217, pp. 188-193.

—., 1991, "Information Is Physical", Physics Today, vol. 44, no. 5, pp. 23-29.

Lewis, David, 1973, "Causation”, Journal of Philosophy, vol. 70, no. 17, pp. $556-567$.

Lombardi, Olimpia, 2005, “Dretske, Shannon's Theory and the Interpretation of Information", Synthese, vol. 144, no. 1, pp. 23-39.

- , 2004, "What Is Information?", Foundations of Science, vol. 9, no. 2, pp. 105-134.

Lombardi, Olimpia, Federico Holik y Leonardo Vanni, 2016, "What Is Shannon Information?", Synthese, vol. 193, no. 7, pp. 1983-2012. 
Lombardi, Olimpia, Sebastián Fortin y Cristian López, 2016, "Deflating the Deflationary View of Information", European Journal for Philosophy of Science, vol. 6, no. 2 pp. 209-230.

Lombardi, Olimpia, Sebastián Fortin y Leonardo Vanni, 2015, “A Pluralist View about Information", Philosophy of Science, vol. 82, no. 5, pp. 1248-1259.

MacKay, Donald, 1969, Information, Mechanism and Meaning, MIT Press, Cambridge, Mass.

— 1956, "Towards An Information-Flow Model Of Human Behaviour", British Journal of Psychology, vol. 47, no. 1, pp. 30-43.

Menzies, Peter y Huw Price, 1993, "Causation as a Secondary Quality", British Journal for the Philosophy of Science, vol. 44, no. 2, pp. 187203.

Nauta, Doede, 1972, The Meaning of Information, Mouton, La Haya.

Nielsen, Michael e Isaac Chuang, 2010, Quantum Computation and Quantum Information, Cambridge University Press, Cambridge.

Pearl, Judea, 2000, Causality, Cambridge University Press, Nueva York.

Penrose, Roger, 1998, "Quantum Computation, Entanglement and State Reduction", Philosophical Transactions of the Royal Society of London $A$, vol. 356, pp. 1927-1939.

Price, Huw, 1991, "Agency and Probabilistic Causality", British Journal for the Philosophy of Science, vol. 42, no. 2, pp. 157-176.

Rovelli, Carlo, 1996, "Relational Quantum Mechanics", International Journal of Theoretical Physics, vol. 35, no. 8, pp. 1637-1678.

Russell, Bertrand, 1948, Human Knowledge: Its Scope and Limits, Simon and Schuster, Nueva York

- 1912, "On the Notion of Cause", Proceedings of the Aristotelian Society, vol. 13, pp. 1-26.

Shannon, Claude, 1993, Collected Papers. ed. N. Sloane y A. Wyner, IEEE Press, Nueva York.

—_, 1948, "The Mathematical Theory of Communication", Bell System Technical Journal, vol. 27, pp. 379-423.

Stonier, Tom, 1996, "Information as a Basic Property of the Universe", Biosystems, vol. 38, pp. 135-140.

Timpson, Christopher, 2013, Quantum Information Theory and the Foundations of Quantum Mechanics, Oxford University Press, Oxford.

- , 2004, "Quantum Information Theory and the Foundations of Quantum Mechanics", tesis doctoral, Universidad de Oxford; disponible en: <https://arxiv.org/pdf/quant-ph/0412063.pdf> [fecha de consulta: $3 / 02 / 2017]$.

Von Wright, Georg, 1971, Explanation and Understanding, Cornell University Press, Ithaca.

Wetzel, Linda, 2009, Types and Tokens: An Essay on Universals, MIT Press, Cambridge, Mass. 
Woodward, James, 2016, "Causation and Manipulability", The Stanford Encyclopedia of Philosophy (edición Invierno de 2016), Edward N. Zalta (ed.); disponible en: <http://plato.stanford.edu/archives/win2013/entries/ causation-mani/ $>$ [fecha de consulta: 3/02/2017].

—_ 2007, "Causation with a Human Face", en H. Price y R. Corry (comps.), Causation, Physics, and the Constitution of Reality: Russell's Republic Revisited, Oxford University Press, Oxford, pp. 66-105.

- - 2003, Making Things Happen: A Theory of Causal Explanation, Oxford University Press, Oxford.

Recibido el 24 de abril de 2017; revisado el 20 de octubre de 2017; aceptado el 24 de noviembre de 2017. 